\title{
An experimental study of regime transitions in a differentially heated baroclinic annulus with flat and sloping bottom topographies
}

\author{
M. Vincze ${ }^{1}$, U. Harlander ${ }^{1}$, Th. von Larcher ${ }^{2}$, and C. Egbers ${ }^{1}$ \\ ${ }^{1}$ Department of Aerodynamics and Fluid Mechanics, Brandenburg University of Technology Cottbus-Senftenberg, \\ Cottbus, Germany \\ ${ }^{2}$ Institute for Mathematics, Freie Universität Berlin, Berlin, Germany
}

Correspondence to: M. Vincze (vincze@tu-cottbus.de)

Received: 31 July 2013 - Revised: 29 November 2013 - Accepted: 15 January 2014 - Published: 21 February 2014

\begin{abstract}
A series of laboratory experiments has been carried out in a thermally driven rotating annulus to study the onset of baroclinic instability, using horizontal and uniformly sloping bottom topographies. Different wave flow regimes have been identified and their phase boundaries - expressed in terms of appropriate non-dimensional parameters - have been compared to the recent numerical linear stability analysis of von Larcher et al. (2013). In the flat bottom case, the numerically predicted alignment of the boundary between the axisymmetric and the regular wave flow regime was found to be consistent with the experimental results. However, once the sloping bottom end wall was introduced, the detected behaviour was qualitatively different from that of the simulations. This disagreement is thought to be the consequence of nonlinear wave-wave interactions that could not be resolved in the framework of the numerical study. This argument is supported by the observed development of interference vacillation in the runs with sloping bottom, a mixed flow state in which baroclinic wave modes exhibiting different drift rates and amplitudes can co-exist.
\end{abstract}

\section{Introduction}

Based on the principle of hydrodynamical similarity, some fundamental characteristics of Earth's various large-scale atmospheric flow phenomena can be modelled using surprisingly simple tabletop-size experimental set-ups. Under laboratory conditions it is possible to control the governing physical parameters and thus to separate different processes that cannot be studied independently in such a complex system as the real atmosphere. Therefore, laboratory experiments provide a remarkable test bed to validate numerical techniques and models aiming to investigate flows observed in the atmosphere and in the oceans.

A classic apparatus to demonstrate the basic large-scale dynamics of the mid-latitude atmosphere is the differentially heated rotating annulus, introduced by Fultz et al. (1959) and Hide (1958), based on the principles suggested by Vettin in the mid-19th century (Vettin, 1857). The set-up (Fig. 1) consists of a cylindrical gap rotating around its vertical axis of symmetry, with a cooled inner side wall and a heated outer side wall, thus the working fluid (usually water or silicone oil) experiences a radial temperature gradient. These boundary conditions imitate the meridional differential incoming solar-heat flux on Earth. The characteristic hydrodynamical timescale scales with the temperature difference, $\Delta T$, between the inner and the outer side wall, and without rotation, the velocity field would be axisymmetric (note, that in such a "sideways convection" configuration, where the heating and cooling takes place at vertical boundaries, there is no minimum $\Delta T$, i.e. any finite temperature difference would initiate an overturning flow.). However, since rotation is present, in the co-rotating reference frame Coriolis' force also acts on the flow, with a magnitude proportional to the flow velocity and the rotation rate, $\Omega$.

The ratio of the revolution period and the aforementioned hydrodynamical timescale yields an appropriate nondimensional number for temperature-driven rotating systems, the thermal Rossby number $R o_{\mathrm{T}}$ (see Sect. 2). For a fluid like water, in case of $R o_{\mathrm{T}} \gg 1$, the flow is axisymmetric and not significantly disturbed by rotation, whereas for $R o_{\mathrm{T}} \ll 1$ (as in the case of cyclones and and anticyclones in the atmosphere) the dynamics is dominated by the 

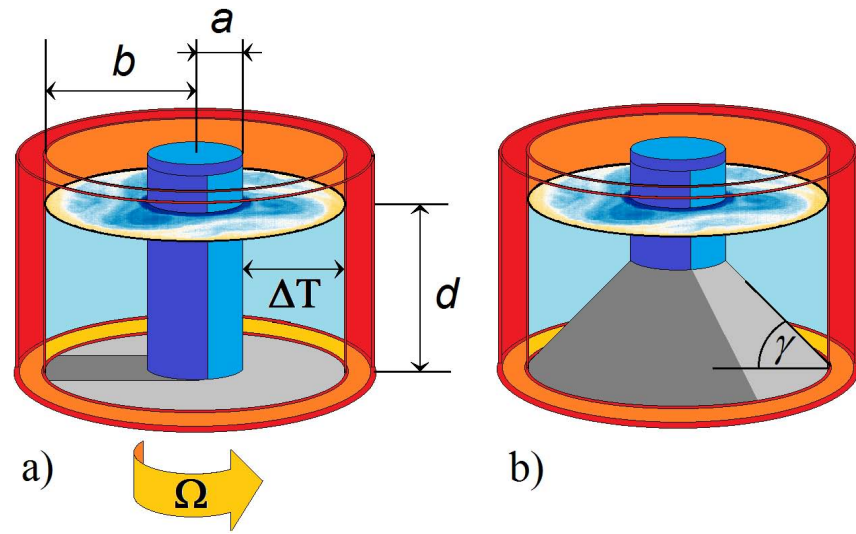

b)

Fig. 1. Schematic drawing of the set-up with a flat (a) and with a sloping-bottom end wall. For the geometric parameters indicated, see text. The anticlockwise direction of rotation is indicated.

Coriolis effect. Another important non-dimensional parameter, the Taylor number, $T a$, can be obtained similarly, by measuring the timescale of viscous effects with respect to the rotation period (more details in Sect. 2). $R o_{\mathrm{T}}$ and $T a$ are used to characterise the different dynamical regimes in the rotating annulus.

Between the axisymmetric and geostrophic turbulent flow state at large Taylor numbers there is a certain region on the $T a-R o_{\mathrm{T}}$ regime diagram, where the velocity and temperature fields exhibit persistent regular wave-like patterns that propagate along the azimuthal direction in the tank due to baroclinic instability (see Fig. 3, or for a more general qualitative view Fig. 1 of von Larcher and Egbers (2005)). The boundary separating the axisymmetric and unstable states on the regime diagram (e.g. the blue and green curves in Fig. 3) is referred to as the "transition curve".

The basic underlying physics of such baroclinic waves has been subject of extensive theoretical (e.g. Eady, 1949; Mason, 1975; Lorenz, 1963), numerical (e.g. Seelig et al., 2012; Read, 1992) and experimental (e.g. Früh and Read, 1997; von Larcher and Egbers, 2005; Harlander et al., 2012) research throughout the past decades. Furthermore, some studies focused on the quantitative comparison of temperature statistics (Gyüre et al., 2007) and propagation dynamics of passive tracers (Jánosi et al., 2010) obtained from annulus experiments and from actual atmospheric data.

The different types of vacillation phenomena (termed "amplitude", "structural" and "interference" vacillation) have attracted considerable attention within the community of geophysical fluid dynamics and of nonlinear systems, dating back to the classic work of Lorenz (1963). The co-existence of different baroclinic wave modes and their interactions in a rotating annulus (with flat bottom) has been the scope of experimental studies, for example, by Pfeffer and Fowlis (1968) or Harlander et al. (2011).
As another example of simple laboratory-scale experiments of geophysical relevance, rotating cylindrical tanks with conical bottom end walls are broadly used as a minimal model of the concept of $\beta$ effect, and in particular to demonstrate topographic (barotropic) Rossby waves, driven by conservation of potential vorticity over a sloping bottom (Rossby, 1949). In a baroclinic set-up, however (i.e. in a system where the isopycnals do not coincide with the equipotential surfaces) the formal analogy between $\beta$ effect and such an end wall configuration does not hold. In this case the introduction of $\beta$ would inherently modify the underlying dynamics, whereas the bottom end wall merely alters the boundary conditions and leaves the equations of motion unchanged. Yet, the sloping differentially heated configuration still provides a useful insight to dispersion effects of baroclinic waves. The wave flow phenomena in a combined differentially heated and sloping set-up have therefore been investigated experimentally, theoretically and numerically to a large extent in the past decades.

To the best of the authors' knowledge, the first in the series of laboratory studies addressing this issue was the work of Fultz and Kaynor (1959), who observed the dispersion of baroclinic waves in the presence of tilted bottom topography, and tried to quantify the group velocities of the propagating patterns. They also compared their results to the then-known dispersion relation of barotropic Rossby waves.

Extensive analytical studies on the dynamics of baroclinic instability over various types of bottom slope have also been published starting from the early 1970s, using various extensions of Eady's model. Within this theoretical framework, Blusmack and Gierasch (1972) have shown that a steep enough bottom slope can fully stabilize the system, once the isopycnals are assumed to be monotonous and uniformly tilted. Mason (1975) further modified Eady's model to incorporate Ekman layer effects and applied it to interpret his experimental results obtained in a baroclinic annular set-up with different bottom and top end wall configurations. Mechoso (1980) extended the model of Blumsack and Gierasch (1972) to include sloping rigid top and evaluated the conditions of stability.

More recently, laboratory experiments have been conducted in a similar configuration by Wordsworth et al. (2008) to elucidate the dynamics of the turbulent regime characterized by large Taylor and low thermal Rossby numbers. There, the sloping boundaries were found to excite turbulent eddies that produce multiple alternating jet structures (also reported by Mason, 1975) via interaction with the zonal mean flow. Similar phenomena have also been detected in internally heated "reversed" annuli with sloping end walls (e.g. Bastin and Read, 1997, 1998). In these set-ups coherent eddies were reported even for steeply sloping boundaries, contradicting the predictions of the aforementioned theoretical models. Similar disagreement has been found and thoroughly discussed by Isachsen (2011) who compared Eady-based and 
numerically simulated tracer diffusivities in baroclinic flows over sloping terrain.

A recent linear numerical study by von Larcher et al. (2013) investigated how the presence of a sloping bottom end wall (combined with a free flat top surface) alters the wave flow regime boundaries, applying the geometrical dimensions of the baroclinic wave tank used for experimental works at the Brandenburg Technical University since about 2003, cf., von Larcher and Egbers (2005). The linear stability analysis of that work revealed significant differences between the flat and sloping bottom end wall configuration. While the overall typical anvil shape of the wave flow regime on the $T a-R o_{\mathrm{T}}$ plane remains similar in the sloping bottom case, the transition curve (more precisely, the neutral linear stability curve) has shifted significantly towards lower values of $T a$, and at higher values of $R o_{\mathrm{T}}$ a sharp reversal of the curve has been found (Fig. 3). The comparison of these numerical results to the actual experiment was the main focus and primary motivation of the present work. We also aimed to obtain a more detailed picture on wave-wave interactions and to gain a deeper insight to the structure of the transition region between the axisymmetric and wave flow regimes.

Our paper is organized as follows. Section 2 outlines the experimental set-up, the parameters that describe the state of the system and the measurement techniques used. Section 3 discusses the applied data evaluation methods. The results are presented in the four subsections of Sect. 4. In Sect. 5 we summarize the results and discuss their implications on the physics of the underlying dynamics.

\section{Experimental apparatus and methods}

Our experiments have been carried out in a circular laboratory tank mounted on a turntable. The tank consisted of three concentric cylinders, rotating at the same rate in anticlockwise direction (at angular velocities between $\Omega=$ $0.13-0.83 \mathrm{rad} \mathrm{s}^{-1}$ ), whose geometric centre coincided with the axis of rotation, as depicted in Fig. 1. In the inner cylinder, made of anodized aluminium, cold water was circulated, the temperature of which was monitored by two thermocouples and adjusted via a cooling thermostat. The "outer ring" of the tank, that is, the annular gap between the two outermost cylinders (painted red and orange in Fig. 1), made of borosilicate glass, hosted the heating wire and contained water as a heat conductive medium. The temperature difference $\Delta T$ between the outer ring and the inner cylinder has been varied between 2.4 and $8.0 \mathrm{~K}$ for the different experimental runs. The methods and characteristics of the temperature control have been discussed in more detail by von Larcher and Egbers (2005).

The middle cavity of the annulus, ranging from $a=4.5 \mathrm{~cm}$ to $b=12 \mathrm{~cm}$ in radial direction (Fig. 1a), was filled up with the working fluid - de-ionized water - to the height of $d=13.5 \mathrm{~cm}$, yielding radius ratio $\eta=a / b=0.38$ and aspect ratio $\Gamma=d /(b-a)=1.8$. The surface of this cylindrical gap was free, which, besides of its importance as boundary condition, also made it possible to use infrared thermography as our measurement technique (infrared radiation is generally absorbed by glass or acryllic, therefore thermography cannot be applied for set-ups with rigid top).

The infrared camera was mounted above the middle of the wave tank and was co-rotating with the set-up. In every $\Delta t=10 \mathrm{~s}, 626 \times 428$ pixel thermograms were taken, covering the surface of the annulus with a resolution of $\sim 0.03 \mathrm{~K}$. The patterns in these thermograms can be considered surface temperature structures, since the penetration depth of the applied wavelength range into water is measured in millimetres.

The most important dynamic control parameters, $\Omega$ and $\Delta T$, can be expressed in terms of non-dimensional numbers. The former is captured by the Taylor number, $T a$, defined as

$T a=\frac{4 \Omega^{2}(b-a)^{5}}{v^{2} d}$,

where $v=1.004 \times 10^{-6} \mathrm{~m}^{2} \mathrm{~s}^{-1}$ is the kinematic viscosity of water. The ratio of the characteristic velocity of the thermally driven flow to the rotation rate yields the thermal Rossby number $R o_{\mathrm{T}}$ (also known as Hide number in honour of Raymond Hide), which serves as a non-dimensional $\Delta T$ scale:

$R o_{\mathrm{T}}=\frac{g d \alpha \Delta T}{\Omega^{2}(b-a)^{2}}$,

where $\alpha=2.07 \times 10^{-4} \mathrm{~K}^{-1}$ is the volumetric thermal expansion coefficient of freshwater and $g$ represents the acceleration due to gravity. Note, that when the horizontal temperature difference $\Delta T$ is comparable to the vertical one, that is, $\Delta T_{z} \approx \Delta T$ holds (a fairly good assumption in the present study), Eq. (2) corresponds to the Burger number $(B)$, defined as the squared ratio of the Rossby deformation radius $R_{\mathrm{d}}=\sqrt{g d \alpha \Delta T_{z}} / \Omega$ to the gap width $b-a$, $B=g d \alpha \Delta T_{z} /\left(\Omega^{2}(b-a)^{2}\right)$.

The physical properties of the working fluid are captured by the Prandl number $P r$ :

$\operatorname{Pr}=\frac{\nu}{\kappa}$,

expressing the ratio of the kinematic viscosity (v) and thermal conductivity $\left(\kappa=0.1434 \times 10^{-6} \mathrm{~m}^{2} \mathrm{~s}^{-1}\right)$ of water (i.e. in our case $\operatorname{Pr}=7.0$ ).

The experiments were conducted the following way: after setting the values of the first target rotation rate and the temperature difference, it took typically around 1.5$3 \mathrm{~h}$ for the temperature control system to reach and stably maintain the required $\Delta T$. The thermographic measurements started when quasi-stationarity of the temperature signals was reached, and lasted for 2000-4000 s, that led to 200-400 snapshots per parameter point. After the first measurement 
was performed, $T a$ was gradually increased or decreased (keeping $\Delta T$ constant) over a period of $1000-2000 \mathrm{~s}$. When the next parameter point was reached, the thermographic measurement started immediately. Thus, the transient flow phenomena could also be recorded, before the system settled its next quasi-equilibrium state. The typical duration scale of this transient phase was 500-1000 s. In one measurement session (typically lasting for $6 \mathrm{~h}$ ) a maximum of 4 different $T a-R o_{\mathrm{T}}$ parameter pairs could be investigated.

The first series of experiments was conducted with flat bottom topography (Fig. 1a), whereas in the second series, a conical sloping bottom obstacle made of polyamide was placed in the cylindrical gap of the tank (Fig. 1b). The slope had an angle of $\gamma=35^{\circ}$ to the horizontal as in the paper by von Larcher et al. (2013), and was decreasing towards the outer side wall, so that the maximum depth $d$ (reached at distance $b$ from the axis) coincided with that in the flat bottom configuration.

\section{Data processing}

In this section we describe the techniques of data evaluation and demonstrate them exemplarily for the reader's convenience using a typical experimental run (with flat bottom end wall) where the flow was dominated by a regular wave pattern, exhibiting 3-fold rotational symmetry.

In order to reduce the investigated parameter space, a path-wise temperature series was extracted from each thermographic image (as the one seen in Fig. 2a). The onedimensional temperature data $T(\theta ; t)$ were obtained along a circular contour at mid-radius $\left(r_{\text {mid }} \equiv(a+b) / 2\right)$ (black circle in Fig. 2a), as a function of the azimuthal angle $(\theta)$, measured clockwise from the "uppermost" point of the circle. The resulting data from this particular snapshot is shown by the black curve of Fig. $2 b$.

For the analysis of the azimuthal temperature fields discrete spatial Fourier transform was used. After subtracting the mean temperature $\langle T(\theta ; t)\rangle$ (averaged over the whole $\theta=[0 ; 2 \pi]$ range at each time instant $t$ ), the remaining fluctuations could be decomposed into amplitudes $A_{m}(t)$ and phases $\phi_{m}(t)$ of normal modes of quantized wave numbers $m=1, \ldots, 6$, as

$T(\theta ; t)-\langle T(\theta ; t)\rangle \approx \sum_{m=1}^{6} A_{m}(t) \cdot \sin \left(m \theta+\phi_{m}(t)\right)$.

Figure $2 b$ demonstrates this step, showing three (exemplarily selected) components: $m=3$ (red), $m=4$ (blue) and $m=6$ (green). The temporal development of the amplitudes of these three modes is presented in Fig. 2c, using the same color encoding. It is clearly visible, that after an initial transient phase, mode $m=4$ has decayed markedly, and from then on the wave pattern was dominated by the leading $m=3$ and $m=6$ modes. After the $t=500 \mathrm{~s}$ mark (vertical dashed
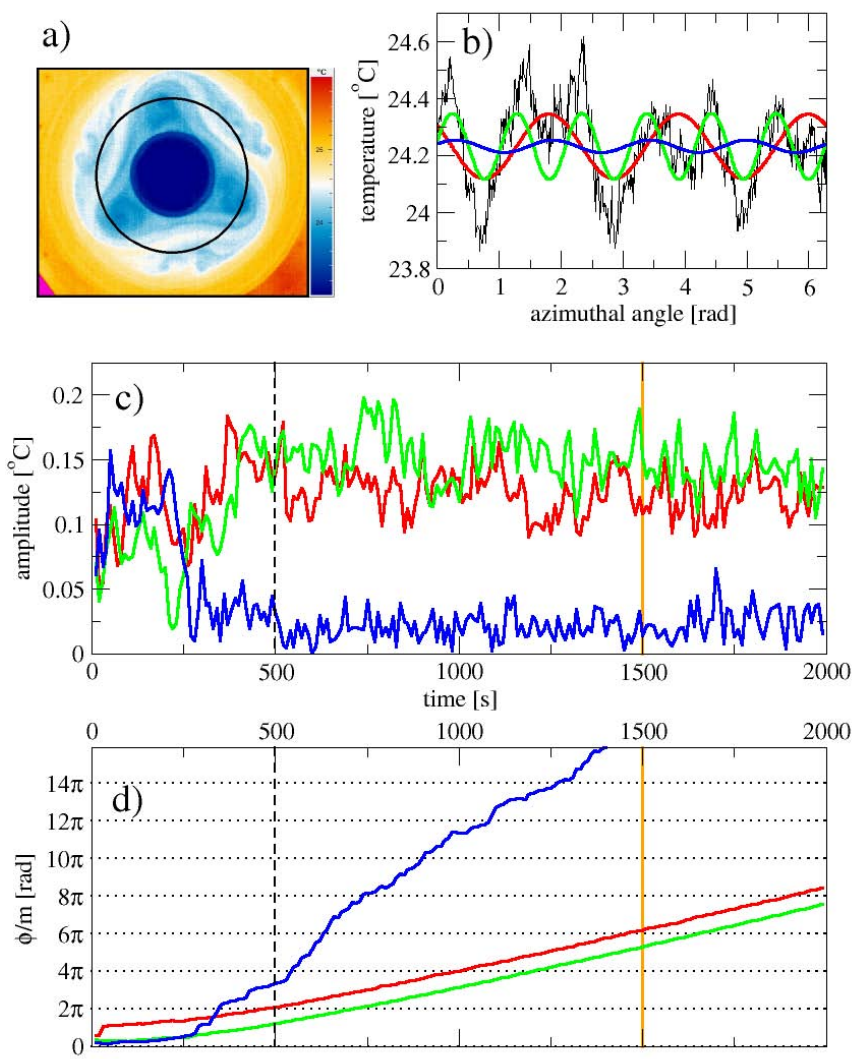

Fig. 2. The steps of data processing. The temperature values from a "raw" thermographic image (a) are extracted along a circular contour (black circle in (a)) to obtain one-dimensional temperature data (b) for spatial Fourier transform. Modes $m=3,4$ and 6 are shown by red, blue and green graphs, respectively. The temporal development of the amplitude (c) and "azimuthal distances" (d) of these spectral components are shown using the same color coding.

line in Fig. 2c and d), the system stayed in a quasi-stationary state (the time instant which corresponds to the patterns of Fig. 2a and $\mathrm{b}$ is marked by an orange vertical line).

Figure $2 \mathrm{~d}$ shows the time series $\phi_{m}(t) / m$ for the above three modes, a quantity that measures the "azimuthal distance" travelled by the given component pattern since $t=0$. For better visualization, we extended the periodical $[0 ; 2 \pi]$ range to $[0 ;+\infty$ ) (so that the positive increments correspond to anticlockwise propagation). This also makes it easier to obtain values for the angular velocities $c_{m}(t)$ of the different modes, since

$\frac{1}{m} \frac{\partial \phi_{m}(t)}{\partial t} \equiv c_{m}(t)$

that is, the values of $c_{m}(t)$ are the slopes of the curves in Fig. 2d. Visibly, modes $m=3$ and $m=6$ propagated at the same rate.

Looking at Fig. 2a and b, it is apparent that $m=3$ is the signature of the general 3-fold symmetry of the wave pattern, whereas the presence of harmonic $m=6$ is related to the 
"fine structure" of the baroclinic wave, most notably to the cold vortices within the three warmer-than-average "lobes". Compared to these, the "azimuthal distance" of $m=4$ exhibited irregular behaviour after its amplitude decayed. In this case, the propagation of $m=4$ was labelled "non-physical". This curve is presented here as an example to demonstrate that such signals could be easily distinguished from the clearly regular drift of the other two modes. This irregular behaviour can be thought of as a "random drift" of the phase $\phi_{m}(t)$. The small amplitude of this component (see Fig. 2c) implies this being "noise" in the Fourier spectra instead of a clear physical signal.

Besides spatial Fourier transforms, standard tools of nonlinear time series analysis have also been applied, some details of which will be briefly discussed in Sect. 4.4.

\section{Results}

Since the present study focused mainly on the transitions between the axisymmetric and the regular wave flow regimes, our experiments were confined to a certain domain of the $T a-R o_{\mathrm{T}}$ parameter space in which such transitions were expected to occur according to von Larcher et al. (2013). Their numerical results are the outcomes of linear stability analyses of non-axisymmetric perturbations - with horizontal wave numbers $m>0$ - applied to the basic axisymmetric $m=0$ state. The latter has been obtained beforehand by iterating the Boussinesq equations using a Fourier-spectral element method. Their curves of neutral linear stability are depicted in Fig. 3 (both for the flat and sloping bottom case). The shaded area denotes the subset of $T a-R o_{\mathrm{T}}$ parameter pairs, that could actually be reached and steadily maintained in our set-up. This constraint is due to the fact that for temperature differences $\Delta T<1 \mathrm{~K}$ the thermal fluctuations coming from technical constraints are almost comparable to the magnitude of $\Delta T$. Therefore, the overlap between the region covered by the numerical study and the one within the reach of our methods is rather small.

With both geometric configurations, the experiments were carried out in the vicinity of the $\Delta T=2.5 \mathrm{~K}$ and $7 \mathrm{~K}$ lines in a domain ranging between $T a=(0.124-4.90) \times 10^{7}$ and $R o=0.55-16.42$. Note, that - as visible in Fig. $3-$ the $\Delta T=7 \mathrm{~K}$ curve already falls out of the range of the numerical runs; here we applied linear extrapolation (dashed blue line in Fig. 3) to approximate the expected regime boundary.

From the alignment of the obtained neutral stability curves - that, by definition, envelope the parameter regime where baroclinic waves are expected to occur - it is possible to formulate a hypothesis to test. If the linear stability analysis would be in itself sufficient to describe the dynamics, one would expect to observe a transition from axisymmetric to regular wave state in the flat bottom configuration at one point of the constant- $\Delta T$ measurement series: the corresponding neutral stability curve (blue) intersects with the

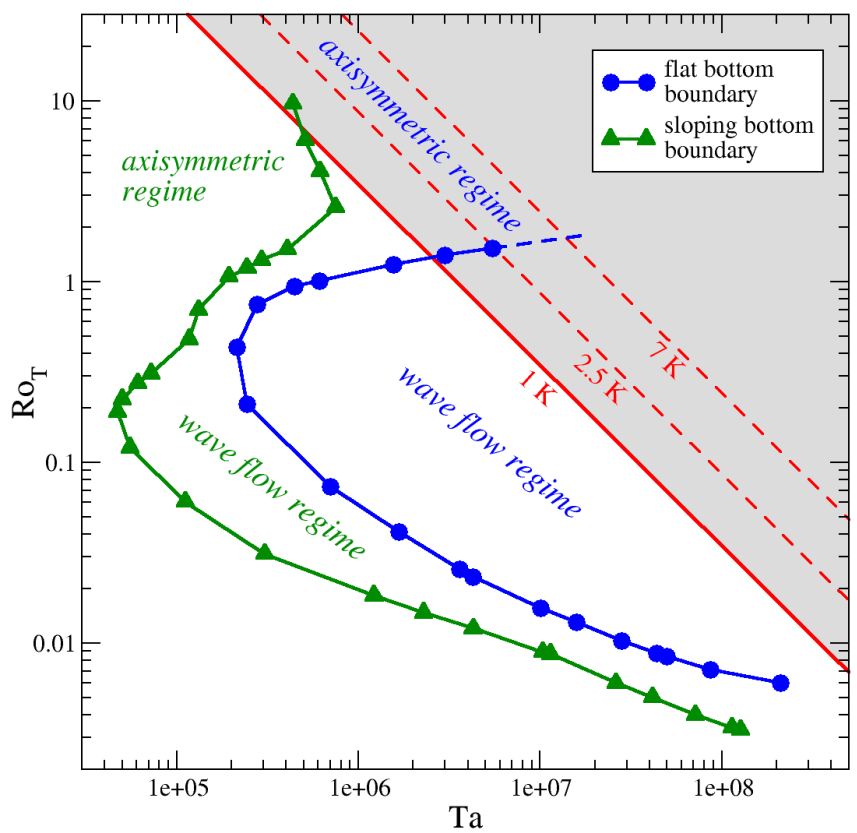

Fig. 3. The neutral stability curves obtained by von Larcher et al. (2013) for flat (blue) and sloping (green) bottom end walls. The lines of constant $\Delta T$ followed by our measurements are also indicated (dashed lines), as well as the parameter region that can be accessed with our techniques (grey shaded area).

lines of constant $\Delta T$ around $R o_{\mathrm{T}} \approx 2$, as seen in the diagram. However, at the same region of the parameter space no such state transition is expected to occur for the sloping arrangement, as the lines of constant $\Delta T$ apparently do not cross the neutral stability curve (green) in this case. Testing this hypothesis has been one of the primary objectives and motivations of the experimental runs described below.

\subsection{Experiments with flat bottom end wall}

Here, we present the results of the first series of experiments, which was conducted with flat bottom topography. Figure 4 shows snapshots of four runs performed in the vicinity of the transition boundary along the $\Delta T=2.5 \mathrm{~K}$ curve, and their respective Hovmöller plots. In agreement with the findings of Früh and Read (1997) and also von Larcher and Egbers (2005), it is visible that between the axisymmetric (AS) flow regime (Fig. 4a) and that of regular propagating wave patterns (Fig. 4c and d) lays a certain transitional range, characterized by dispersive weak waves (hereafter denoted with "WW") of fluctuating wave amplitudes (Fig. 4b). The diagram of the flat bottom measurements is presented in Fig. 5. In the $\Delta T=(7-8) \mathrm{K}$ region, data points obtained from earlier experimental studies using the same configuration (Seelig et al., 2012; Harlander et al., 2011) were also adopted.

The existence of multiple equilibria (i.e. different flow types at the same values of $T a$ and $R o_{\mathrm{T}}$ ) is apparent from the regime diagram, indicated by the fact that the region "WW" 

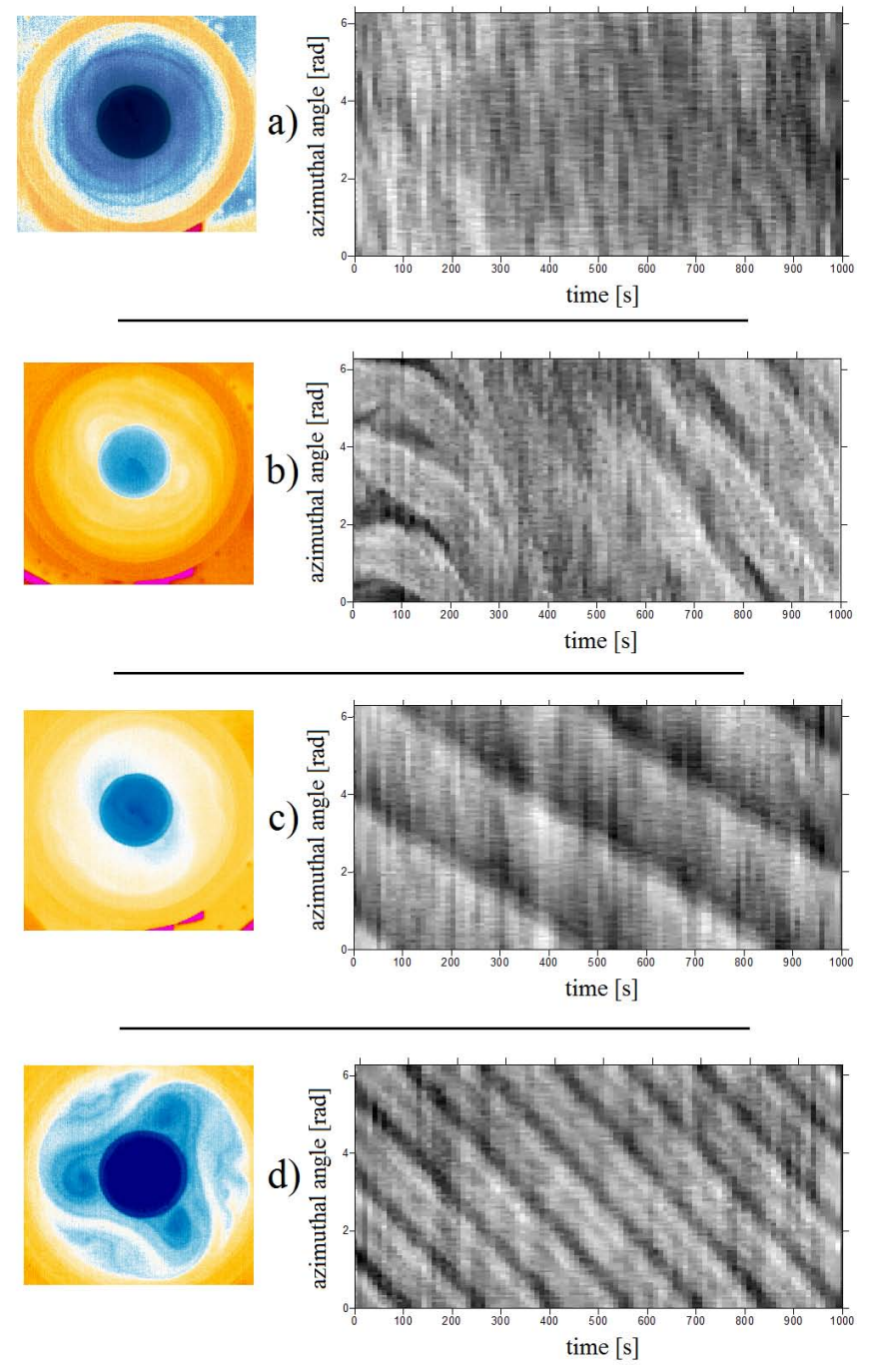

Fig. 4. Thermographic snapshots (left) and Hovmöller plots (right) of some experimental runs with flat bottom topography. (a) Axisymmetric $(m=0)$ basic state $\left(R o_{\mathrm{T}}=2.73 ; \mathrm{Ta}=3.31 \times 10^{6}\right)$, (b) dispersive fluctuating weak wave (WW) state with traces of modes $m=2,3\left(R o_{\mathrm{T}}=2.43 ; \mathrm{Ta}=3.60 \times 10^{6}\right)$, (c) regular wave with $m=2$ being the dominant component $\left(R o_{\mathrm{T}}=1.98 ; \mathrm{Ta}=\right.$ $\left.4.59 \times 10^{6}\right)$, and $(\mathbf{d})$ regular pattern with leading component $m=3$ $\left(R o_{\mathrm{T}}=1.55 ; \mathrm{Ta}=5.78 \times 10^{6}\right)$.

of weak fluctuating waves visibly overlaps with the axisymmetric regime in Fig. 5. These damped waves of the "WW" regime can be interpreted as the signs of the extreme sensitivity of the system to small external perturbations in this transient zone. Non-modal transient growth of small fluctuations may be relevant in this regime, as discussed by Seelig et al. (2012). Occasionally non-unique states emerged as well, in which two given regular wave modes of different wave numbers (typically $m=2$ and $m=3$ ) were alternating, replacing each other on a typical timescale of $\sim 10$ revolutions. This kind of dynamics is referred to as "intermittent

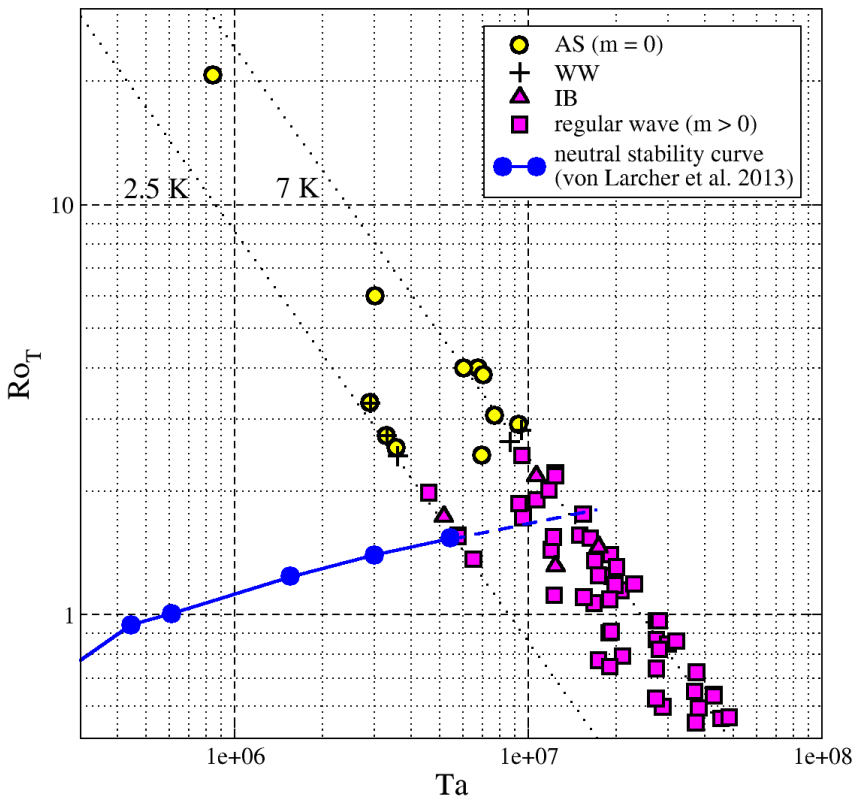

Fig. 5. The regime diagram for the flat bottom experiments. The neutral numerical stability curve is marked by the blue solid curve (extended as a dashed line for the region not covered by von Larcher et al. (2013)). Yellow circles indicate axisymmetric (AS) wave flow, plus signs show data points where weak wave (WW) propagation was observed, upward triangles and squares indicate intermittent bursting (IB) and regular wave flow, respectively.

bursting" (IB), and has already been reported by von Larcher and Egbers (2005) (see also references therein).

The region where the transition from the axisymmetric state to the wave flow regime occurs shows qualitative agreement with the computed neutral linear stability curve (blue graph in Fig. 5) obtained by von Larcher et al. (2013). The azimuthal wave number $m=2$ of the first regular wave pattern that appeared in the experiment after the transition (e.g. Fig. 4c) is also consistent with the first unstable mode of the numerical analysis in this parameter regime. We emphasize, however, that a sharp transition curve cannot be determined for the experiments, since the intermediate transient "WW" state forms a rather smoothly changing continuum between the AS and the steady wave regime.

\subsection{Experiments with sloping bottom end wall}

We now present our findings in the sloping bottom case. With the introduction of sloping bottom topography, a certain type of vacillation was observed which did not appear in the flat bottom case. The steady wave patterns that occur regularly in the flat bottom case (see Hovmöller plots in Fig. 4c and d) have not been observed in any of our sloping bottom experiments. Instead, the dominant baroclinic wave mode appeared in distorted form, modulated periodically by another wave of different wave number and propagation rate. This superposition phenomenon is illustrated by the snapshots and 

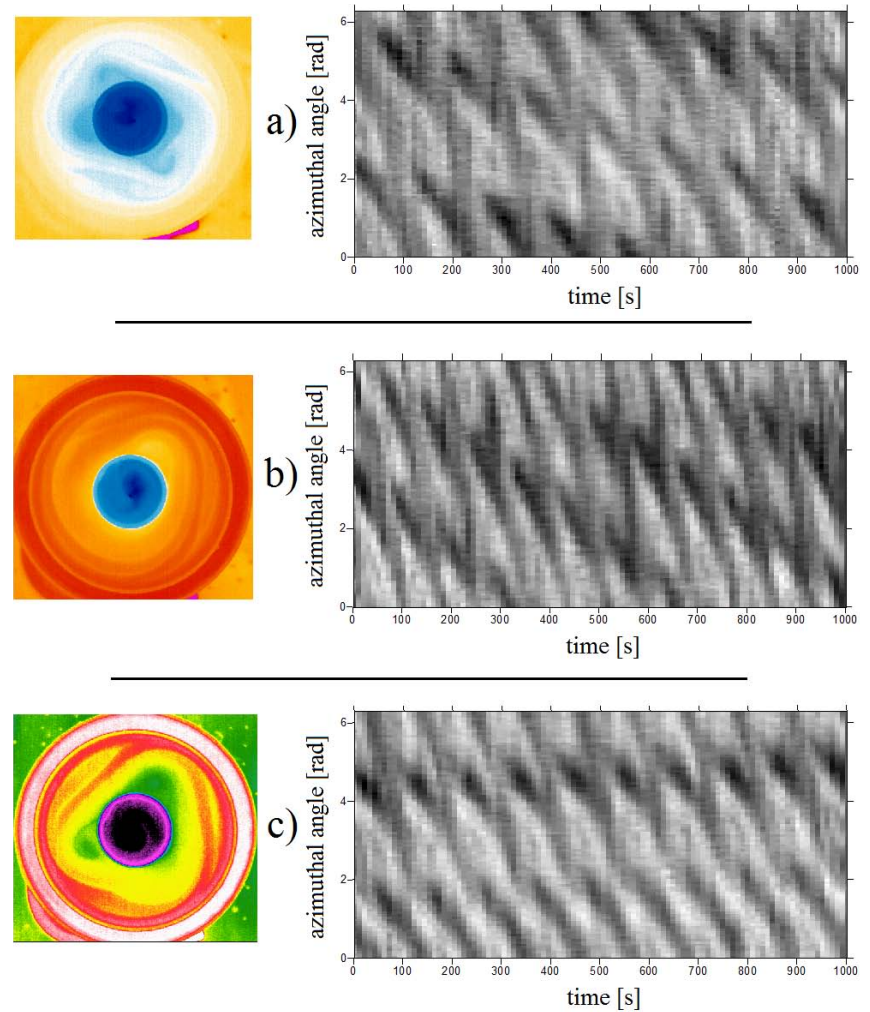

Fig. 6. Thermographic snapshots (left) and Hovmöller plots (right) of three experimental runs exhibiting interference vacillation flows with sloping bottom topography. (a) Involved modes: $m=2,3,5$ $\left(R o_{\mathrm{T}}=1.59 ; \mathrm{Ta}=6.04 \times 10^{6}\right),(\mathbf{b})$ involved modes: $m=3,2,1$ $\left(R o_{\mathrm{T}}=1.53 ; \mathrm{Ta}=1.35 \times 10^{7}\right),(\mathbf{c})$ involved modes $m=2,3,5$ $\left(R o_{\mathrm{T}}=1.10 ; \mathrm{Ta}=1.84 \times 10^{7}\right)$. Note, that unlike in Fig. 4 , the contributing wave numbers are not that manifest from pure visual inspection of the plots. The involved modes were determined using the methods described in Sect. 4.3.

Hovmöller plots of Fig. 6. The observed mechanism is referred to as interference vacillation (IV), cf. Lindzen et al. (1982).

It is generally accepted that the presence of $\beta$ effect enhances dispersion of baroclinic waves, as it was already demonstrated experimentally in a similar set-up (including sloping bottom topography) in the paper by Fultz and Kaynor (1959).

As an example, a clear signature of dispersion is presented in Fig. 7, for an experiment performed at $T a=2.23 \times 10^{7}$, $R o_{\mathrm{T}}=0.93$. Figure $7 \mathrm{a}$ shows the temporal development of the spatial Fourier amplitudes of components $m=3,4$ and 6, whereas in Fig. 7b the spatial propagation of these modes is shown (cf. Fig. 2c and d for flat bottom). As visible in Fig. 7a, during the initial transient "spin-up" phase, mode $m=4$ dominated the flow. This component later decayed in amplitude, transferring its energy to the $m=3$ mode and its $m=6$ harmonic (which is the fingerprint of the fine structure of the baroclinic wave, as noted in Sect. 3). Yet, a
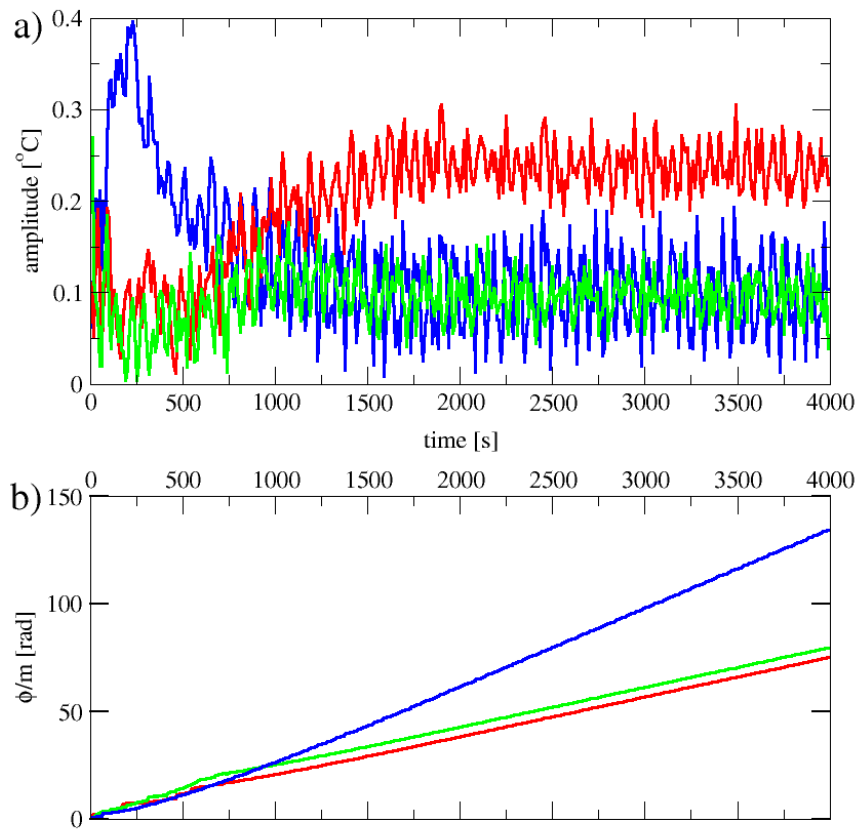

Fig. 7. The temporal development of the amplitudes (a) and "azimuthal distances" of modes $m=3$ (red), $m=4$ (blue) and $m=6$ (green) for an experimental run with sloping bottom $(T a=2.23 \times$ $10^{7}$ and $\left.R_{\mathrm{T}}=0.93\right)$.

perturbation that inherited the 4-fold symmetry of the transient initial state did not vanish, but continued to propagate at a significantly larger phase velocity than that of the dominant mode (see Fig. 7b).

It is worth mentioning that the phase speed of a given mode generally increased as its amplitude decreased. This is visible from the comparison of Fig. 7a and b: the propagation of $m=4$ clearly accelerated until $t \approx 700 \mathrm{~s}$ and then stayed constant (see blue curve Fig. 7b), meanwhile its amplitude showed the aforementioned decay until the same $t \approx$ 700 s mark, and exhibited stationary behaviour afterwards (see blue curve in Fig. 7a). Qualitatively similar amplitude vs. phase speed relations could be observed in the temporal development of the other modes as well. These findings underline the nonlinear property of the dynamics present in the baroclinic annulus.

We summarize our findings for the sloping bottom case in the regime diagram of Fig. 8. In these experiments IV wave patterns have been observed in the region of the $T a-R o_{\mathrm{T}}$ plane which was characterized by regular steady waves in the flat bottom case. However, the overall structure of the transition region remained quite similar to the one of Fig. 5 (the flat bottom neutral stability line is marked by a dashed blue line in Fig. 8). This result shows that in this geometry (in contrast with the flat bottom case) the actual regime transition does not occur along the neutral stability curve (green) calculated by von Larcher et al. (2013). The disagreement between the linear stability analysis and the actual flow (as 


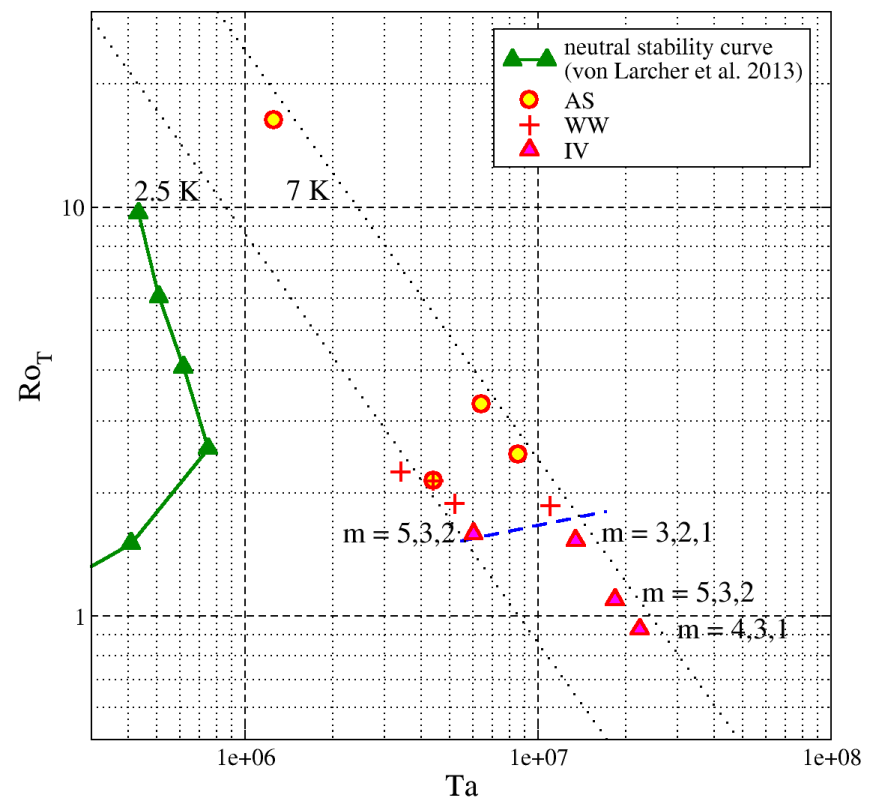

Fig. 8. Regime diagram for the sloping bottom experiments. The neutral numerical stability curve for this case is marked by the green solid curve. Circles indicate axisymmetric (AS) wave flow, plus signs show data points where weak wave (WW) propagation was observed, whereas upward triangles indicate interference vacillation (IV) flow. For the data points exhibiting IV, the wave numbers of the interacting wave triads are indicated.

well as the presence of IV) implies nonlinear wave-wave and wave-background flow interactions that are enhanced by the sloping bottom. As visible in Fig. 8, the neutral linear stability curve exhibits a sharp reversal at higher values of $R o_{\mathrm{T}}$, as well as a general destabilizing effect of the sloping bottom end wall, which manifests itself in the fact that the curve of the sloping case envelopes that of the flat bottom case (see also Fig. 3). Thus, without nonlinear interactions, regular wave propagation would be observed all over the experimentally investigated parameter region in the sloping bottom configuration.

\subsection{Mode identification in the IV regime}

The simplest form of wave-wave interactions is a three-wave resonance, in which two coexisting waves of different wave numbers and drift rates excite and amplify a third mode (e.g. Plumb, 1977). The selection criteria of such resonant triads are that the wave vectors $\boldsymbol{k}_{i}$ of the modes involved should add up to zero, as

$\boldsymbol{k}_{m} \pm \boldsymbol{k}_{m^{\prime}} \pm \boldsymbol{k}_{m^{\prime \prime}}=0$,

and also the following relationship should hold between the spatial phases $\left(\phi_{i}\right)$ of the three interacting modes (Früh and Read, 1997):

$\phi_{m}(t)-\phi_{m^{\prime}}(t)-\phi_{m^{\prime \prime}}(t) \equiv \varphi_{m-m^{\prime}-m^{\prime \prime}}(t) \approx$ const.

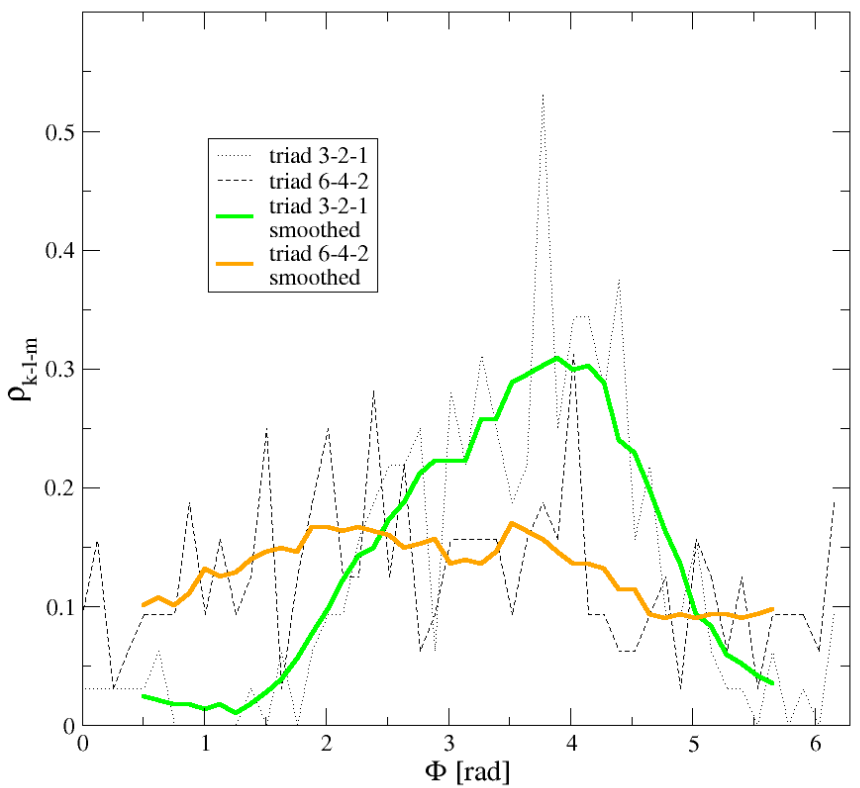

Fig. 9. Triad locking probability density functions for triads $m=3,2,1$ and $m=6,4,2$, and their (9-point) moving-averaged, "smoothed" versions in a sloping bottom experiment. See also the legend. $\left(\right.$ Ro $\left.\mathrm{T}=1.53 ; \mathrm{Ta}=1.35 \times 10^{7}\right)$.

For an exactly resonant situation, termed "phase lock", the time series $\varphi_{m-m^{\prime}-m^{\prime \prime}}(t)$ would give a constant value between 0 and $2 \pi$. One can therefore define a probability density function $\rho_{m-m^{\prime}-m^{\prime \prime}}(\phi)$ of $\varphi_{m-m^{\prime}-m^{\prime \prime}}(t)$, that practically means a histogram of the time series. If marked phase coherence (i.e. triad resonance) is present, then $\rho_{m-m^{\prime}-m^{\prime \prime}}(\phi)$ should exhibit a sharp peak, whereas, if Eq. (7) was not satisfied, the distribution would be rather flat.

In terms of the azimuthal wave numbers, studied throughout this paper, Eq. (6) can simply be formulated as

$m \pm m^{\prime} \pm m^{\prime \prime}=0$.

As mentioned in Sect. 3, our Fourier transform scheme was based on components of integer wave numbers $m=1$ to 6 . Among these, five triad combinations can be selected that fulfil Eq. (8). In order to identify the most dominant interacting waves, the density functions $\rho_{m-m^{\prime}-m^{\prime \prime}}(\phi)$ of each triad were analysed for all four experiments where IV was detected. Two examples of $\rho_{m-m^{\prime}-m^{\prime \prime}}(\phi)$ are shown in Fig. 9. In this particular case $\rho_{3-2-1}(\phi)$ exhibits a well-defined peak, whereas $\rho_{6-4-2}(\phi)$ does not show any significant coherence (see also the corresponding Hovmöller plot and the parameters in Fig. 6b).

The analysis of the triad locking density functions combined with that of the amplitude and phase time series (e.g. the ones in Fig. 7) enabled us to identify the most dominantly interacting modes in all four experiments where IV was detected. The resulting wave numbers of the dominant triads are marked in the regime diagram of Fig. 8. 
a)

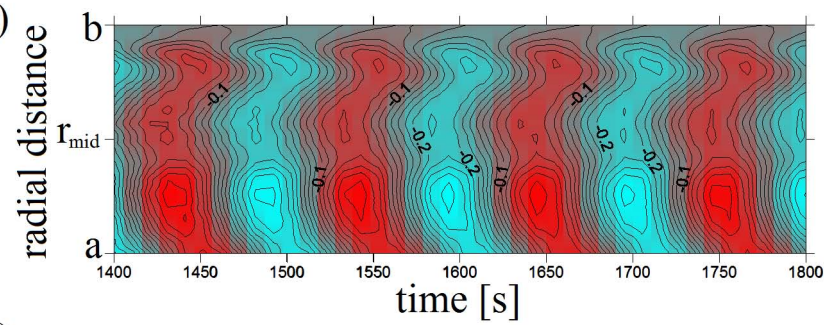

b)

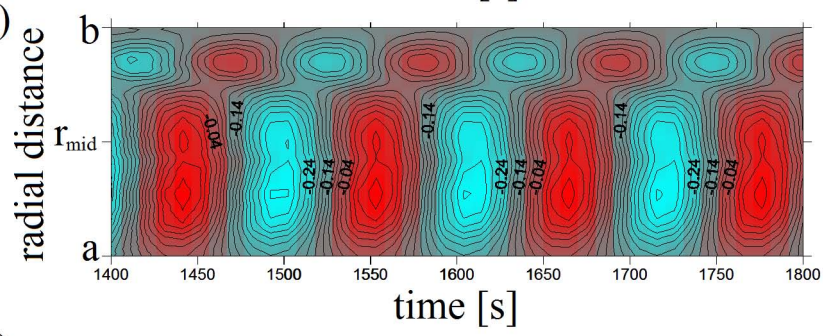

c)

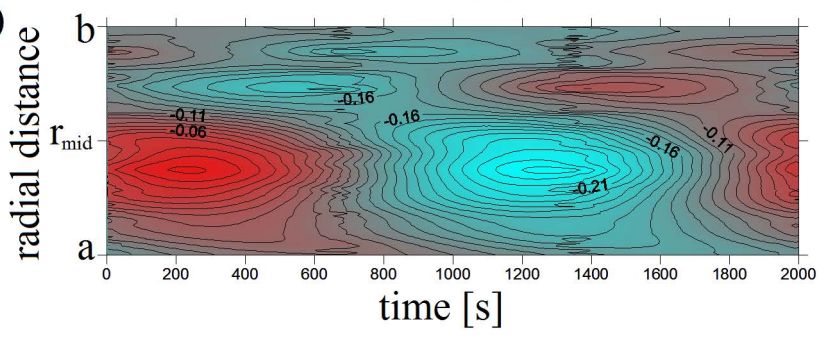

Fig. 10. Hovmöller plots of the filtered radial patterns that correspond to the frequencies of the azimuthal wave motions the triad of $m=3$ (a), $m=5$ (b) and $m=2$ (c). Red colours denote regions of higher temperatures. The contour spacing was set to $0.1 \mathrm{~K}$. Note the wider temporal range in c) $\left(R o_{\mathrm{T}}=1.099 ; \mathrm{Ta}=1.83 \times 10^{7}\right)$.

Although this investigation focused on the azimuthal wave numbers, it is to be noted that the relation Eq. (8) is a necessary but per se not a sufficient condition to fulfil the selection criterion Eq. (6) of the wave vectors. Equations analogous to Eq. (8) exist for the radial $(l)$ and vertical $(n)$ components of the triad-forming wave vectors, in the form of

$l \pm l^{\prime} \pm l^{\prime \prime}=n \pm n^{\prime} \pm n^{\prime \prime}=0$.

Since the vertical behaviour could not be directly detected with our measurement techniques, we analysed the radial patterns corresponding to the interacting azimuthal modes. To reveal these structures, path-wise temperature series were extracted from each thermographic frame (as described in detail in Sect. 3), this time along a radial section ranging from $a$ to $b$ and consisting of 110 grid points at each time instant. As the next step, bandpass filtering has been applied to all of these 110 temperature records in the (disjoint) $f_{m}=m \cdot c_{m} /(2 \pi) \pm 1.5 \times 10^{-4} 1 / \mathrm{s}$ frequency ranges, taken with the angular velocities $c_{m}$ of the azimuthal modes $m$, obtained from Eq. (5). Then the filtered time series were recombined into Hovmöller plots like the ones presented in Fig. 10 as typical examples. These show the radial wave motion associated with the frequencies of the dominant interacting

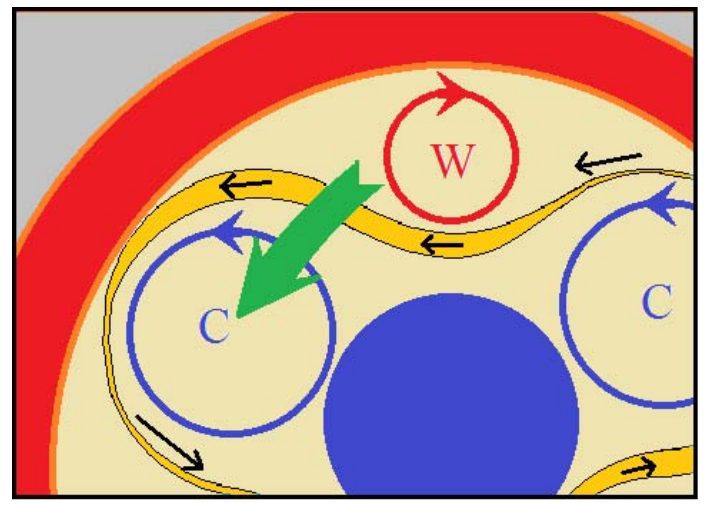

Fig. 11. Schematic draft of the spatial asymmetries between the front and the wake sides of a baroclinic lobe. Note that the jet (orange), meandering between cold (C) and warm (W) vortices accelerates (see arrows) and therefore compresses at the front side, compared to the wake side.

triad ( $m=5,3$ and 2$)$ of the experimental run at $R o_{\mathrm{T}}=1.099$ and $T a=1.83 \times 10^{7}$.

Visibly, the patterns in Fig. 10a $(m=3)$ and Fig. 10b $(m=5)$ are more complex than pure standing wave modes characterized by a single value of $l$, yet a regular, spatially correlated structure is present. In Fig. $10 \mathrm{~b}$ even a steady node can be observed at radial distance $r \approx\left(r_{\text {mid }}+b\right) / 2$ which separates an inner domain from an outer one, oscillating at a clear phase lag of one-fourth of a period. These Hovmöller plots indicate that even in these narrow frequency bands $\left(f_{m}\right)$ the radial dynamics is driven by the superposition of at least two standing wave modes, some of which fulfil Eq. (9), while others do not.

\subsection{Time-reversal asymmetry and traces of nonlinearity}

It has been generally observed that in the regular, nondispersive baroclinic waves of the flat bottom configuration (e.g. the one in Fig. $4 \mathrm{~d}$ a jet is formed along the surface streamlines, encompassing the "cold" eddies (see orange strip in the schematic drawing of Fig. 11). As the wave pattern drifts (green arrow), the streamlines at the front of the cold lobes compress (the jet accelerates) and thus the temperature gradient steepens ("nonlinear steepening"). Meanwhile, in the wake of the cold vortices the gradient flattens. This spatial asymmetry, a sign of nonlinear wave propagation, manifests itself as the time-reversal asymmetry of the temperature time series obtained from co-rotating temperature measurements. A similar asymmetry has been reported by Gyüre et al. (2007) in the regime of geostrophic turbulence as well, in experiments with flat bottom end wall. In the sloping bottom case, however, this structure is expected to be distorted by the wave-wave interactions. The modulation caused by the other wave mode(s) involved in the interference vacillation destabilizes the jet and leads to mixing, which may reduce the asymmetry, thus providing 


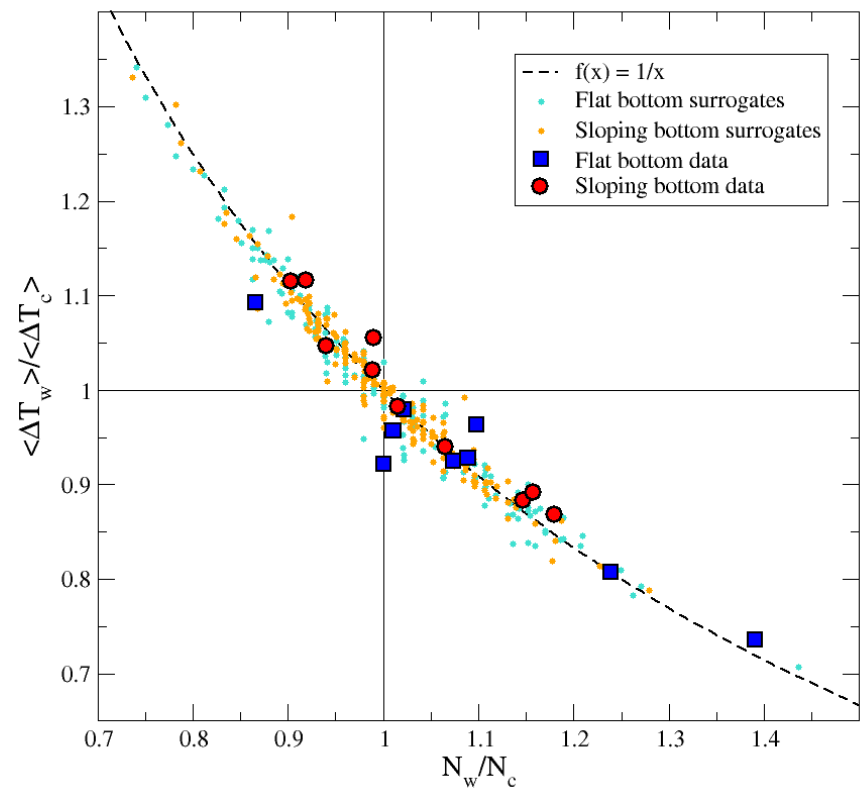

Fig. 12. Correlation plot between step number ratios and average step size ratios from surface temperature records for both bottom end wall configurations. The values corresponding to the "shuffled" surrogate time series are also presented. Dashed line denotes $1 / x$. See also the legend.

further evidence for the existence of wave-wave interactions. This hypothesis has been tested by analysing pointwise surface temperature records from our experiments for both geometries.

The time series were extracted from the same raw thermographic images as the azimuthal temperature field data, after selecting a location at radial distance $r_{\text {mid }} \equiv(a+b) / 2$. Naturally, the sampling time was the same as mentioned in Sect. 3, i.e. $\Delta t=10 \mathrm{~s}$. In case of data loss (which may occur, e.g. because of the occasional automatic recalibration of the microbolometers in the infrared camera), linear interpolation was applied to yield equally spaced data.

The aforementioned temporal asymmetry has been quantified in terms of two simple quantities by Gyüre et al. (2007): the ratio of the number of warming and cooling steps $\left(N_{\mathrm{w}} / N_{\mathrm{c}}\right)$ in the time series of a given experimental run, and the ratio of their mean magnitudes, averaged over the record, $\left\langle\Delta T_{\mathrm{w}}\right\rangle /\left\langle\Delta T_{\mathrm{c}}\right\rangle$. The significant deviation of these parameters from unity (i.e. the presence of temporal asymmetry in stationary time series) can be interpreted as a sign of nonlinearity, as mentioned above. The same type of analysis has been repeated in the present study.

Similarly to Gyüre et al. (2007), to define a $95 \%$ confidence interval, 20 random shuffled time series were produced from the original signals of the experimental runs, for each record. In order to achieve this, the iterative Fourier surrogate method by Schreiber and Schmitz (2008), and the opensource software package TiSeAn 3.0.1 (by the same authors) were applied. Besides conserving the histogram, this procedure also keeps Fourier amplitudes intact (by shuffling only the phases of the spectral components), but destroys all nonlinear correlations in the signal. The surrogate data produced this way should theoretically exhibit full symmetry (i.e. both $\left.N_{\mathrm{w}} / N_{\mathrm{c}}=\left\langle\Delta T_{\mathrm{w}}\right\rangle /\left\langle\Delta T_{\mathrm{c}}\right\rangle \equiv 1\right)$, yet, due to the finite length of the time series, certain deviations are present. However, the distribution of the parameter values for these shuffled signals is symmetric to 1 , as shown by the small turquoise and orange dots in the scatter plot of Fig. 12, representing the values obtained for the flat and sloping bottom surrogates, respectively.

If the conditions of stationarity are fulfilled, the above defined two measures of asymmetry should be inversely proportional to each other; the smaller is the number of steps in one direction (compared to that of the other direction), the larger the average relative size of the magnitude of these steps should be, to avoid increasing or decreasing trends. Thus, the fact that all the data points of Fig. 12 - both from surrogates and actual signals - are scattered along the $f(x)=$ $1 / x$ curve (dashed line) implies that stationarity holds for our experimental data.

The most important observation is that the scattering of the data points obtained from the experiments with flat bottom end wall (blue squares in Fig. 12) shows a marked deviation from unity (and from the distribution of the surrogates), and is clearly asymmetric. For the vast majority of the realizations $N_{\mathrm{w}} / N_{\mathrm{c}}>1$ and $\left\langle\Delta T_{\mathrm{w}}\right\rangle /\left\langle\Delta T_{\mathrm{c}}\right\rangle<1$ can be found, indicating nonlinearity. We note, that the extent and direction of this asymmetry obtained close to the axisymmetric regime is in good agreement with the findings of Gyüre et al. (2007), although, as mentioned above, their experiments were performed in the turbulent regime (at $\mathrm{Ta}=9.43 \times 10^{9}$ and $R o_{\mathrm{T}}=0.035$ ). As expected, for the sloping bottom experiments (red circles in Fig. 12) no such tendency was observed; the distribution of these data points is fairly symmetric with respect to 1 and overlaps with that of their surrogates. This result confirms our assumption that wave-wave interactions in the sloping bottom experiments generally destroy the structure of the jet encompassing the cold lobes and thus lead to the reduction of steepening and to the homogenization of the flow field.

\section{Discussion}

Two series of laboratory experiments have been performed in a differentially heated rotating annulus set-up, recorded by a co-rotating high precision infrared camera and evaluated using digital image processing techniques, spatial Fourier transforms and standard tools of nonlinear time series analysis. The most important motivation for these measurements was to compare the alignment of the boundary between the axisymmetric and the regular wave flow regimes on the parameter space spanned by the Taylor and thermal Rossby 
numbers with the curve of neutral linear stability, obtained in a numerical study by von Larcher et al. (2013).

In the first series of experiments the bottom end wall of the tank was flat. As far as this geometry is concerned, our experimental results showed a qualitative agreement with the numerical results. It was pointed out, that between the axisymmetric state and the domain of robust regular baroclinic waves a transient behaviour can be observed, characterized by uncertain, fluctuating wave patterns. The occurrence of such transient flows makes it difficult to define a sharp transition from the axisymmetric to the wave state. This explains why we find qualitative agreement only. The transient regime exhibits multiple equilibria: vacillating decaying waves and axisymmetric flow can both exist at the same values of $T a$ and $R o_{\mathrm{T}}$. Because of their short lifespan and fluctuating amplitudes, no conclusive dispersion relation of these waves could be obtained, but from the visual inspection of Hovmöller plots as the one in Fig. 4b, it can certainly be stated that the drift rates of these waves clearly depend (among other things) on the wave number (i.e. dispersion is present). Once the stable wave flow regime is reached, dispersion ceases to exist; all the (physically meaningful) Fourier components propagate at the same rate, maintaining the complex pattern of the steady baroclinic waves.

The second series of measurements was performed with a sloping bottom placed in the annulus. The key issue that has been addressed in this case was whether this modification stabilizes or destabilizes the system. The classic theoretical approach to this problem, for example, the one followed by Mason (1975), is based on an extension of the classic baroclinic instability theory of Eady (1949) to include Ekman layers. Mason obtained regime diagrams for different top and bottom end wall configurations, including ones similar to the cases studied here. In Fig. 14 we repeat Figs. 20 and 21 of his original paper, overlaid onto each other to highlight the differences. The stability parameter $(T o)$, defined as the ratio of the bottom slope to the slope of the isopycnals, has the value of $T o=0.327<1$ in the sloping case of Fig. 14. Note, that this value as well as the geometric properties of the annulus described there differ from those of the one used in our study, and so are the control parameters. Yet, parameter $\mathcal{T}$ of the horizontal axis is equivalent to $T a$ and the Burger number $B$ of the vertical axis is proportional to $R o_{\mathrm{T}}$ (as the model assumes parallel, uniformly tilted isopycnals), thus these curves are suitable for qualitative comparison with Fig. 3. The main distinctive feature of the regime diagram for the sloping case (blue graph in Fig. 14) compared to that of the flat bottom case (red contour) is that the "lower axisymmetric" regime has been destabilized. On the other hand, the boundary between the wave flow regime and the "upper" axisymmetric region remains basically unaffected. We note, that our ongoing numerical simulations with the same code as used by von Larcher et al. (2013) for an intermediate bottom slope of $14^{\circ}$ shows no sharp reversal of the linear stability curve (in contrast to the green curve of Fig. 3).
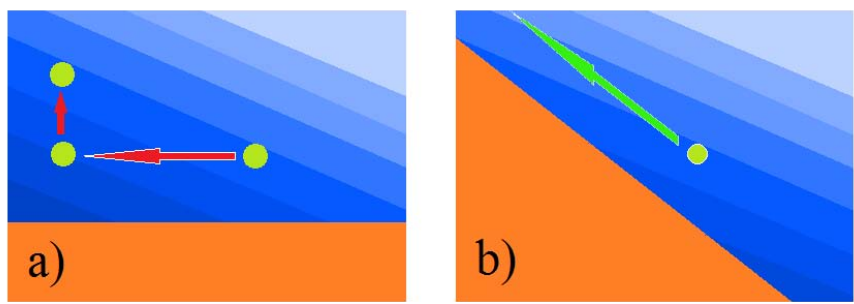

Fig. 13. Schematic drawing of the qualitative background of baroclinic instability. For a flat bottom topography (a) a small horizontal displacement of a fluid parcel leads to a larger displacement, i.e. instability occurs. If the bottom topography is steep enough (b), geometric constraints inhibit destabilization in the bottom region.

Instead, at large values of $R o_{\mathrm{T}}$ the neutral stability line follows the curve, in agreement with Mason (1975). Surprisingly, these two analyses (both obtained for $T o<1$ ) seem to be consistent with our experimental findings, although, based on a simple order-of-magnitude estimate (see later), one would expect that for our case generally $T o>1$ holds.

According to Eady model-based linear stability analyses (see, e.g. Blusmack and Gierasch, 1972; Mechoso, 1980) for $T o>1$ the flow is expected to be completely stabilized. The schematic drawing of Fig. 13 illustrates this argument. In the "standard" case of baroclinic instability, a fluid parcel which is moved by some initial perturbation parallel to the (flat) bottom, though travelling along an equipotential, may reach a region of higher density, since the thermal wind balance keeps the isotherms tilted (Fig. 13a). From here, the buoyancy force lifts the parcel until it reaches its original isotherm - at a larger geopotential. The increased potential energy of the system is responsible for the excitation of baroclinic waves. If, however, the slope of the bottom is steeper than that of the isothermal surfaces, a parcel moving parallel to the bottom is pushed back to its initial position by the buoyancy, therefore no instability can arise in the bottom region (Fig. 13b).

Within the framework of the geostrophic theory of thermal wind, one can easily obtain an order-of-magnitude estimate of the slope $s_{T}$ of isotherms (see, e.g. Pedlosky, 1979) in the form of

$s_{T}=\frac{f \cdot U}{g \alpha \Delta T}$,

where $U$ denotes the characteristic velocity of the zonal background flow (the other symbols denote the same quantities as in Eq. 2). Fortunately, there exist earlier PIV (particle image velocimetry)-based measurement data on the velocity $U$ from the same $T a-R o_{\mathrm{T}}$ parameter range and the same geometry - albeit for flat bottom only - as reported by Harlander et al. (2011). Based on these data, we can take $U \sim 10^{-3} \mathrm{~m} \mathrm{~s}^{-1}$ as a safe (over-) estimation of the background flow. In the same spirit, we can substitute the smallest applied temperature difference $\Delta T=2.45 \mathrm{~K}$, as well as the 


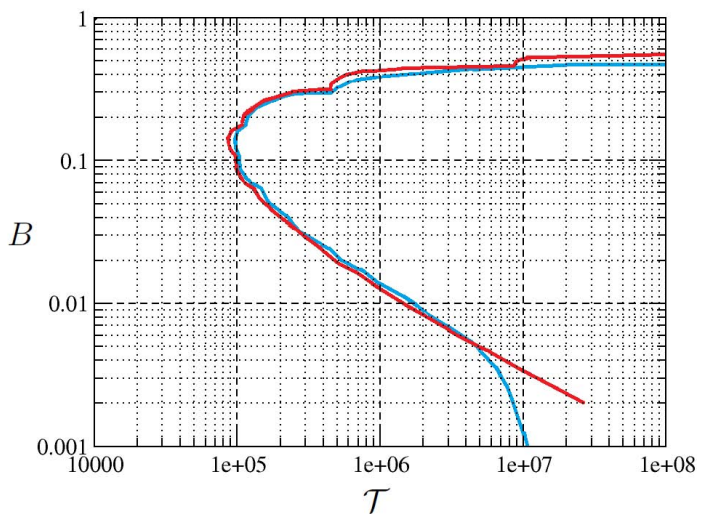

Fig. 14. Regime diagrams obtained by Mason (1975) for flat (red) and gently sloping (blue) bottom end walls, cf. Fig. 3.

largest Coriolis parameter $f=2 \Omega=1.12 \mathrm{~s}^{-1}$ into Eq. (10), which yields $s_{T}^{\max } \approx 0.2$, still significantly smaller than the slope $\tan (\gamma)=0.7$ of our bottom topography, yielding slope ratio $T o \equiv \tan (\gamma) / s_{T} \approx 3.5>1$. Therefore, based on this argument, one would expect that the instability is inhibited by the slope, and thus the wave flow regime in the $T a-R o_{\mathrm{T}}$ plane would considerably shrink compared to the flat bottom case.

The fact that neither the experiments, nor the numerical simulations of von Larcher et al. (2013) showed such stabilization may be explained by the fact that the slopes of the isotherms are clearly not independent from the bottom topography - as assumed in Eq. (10) - but instead, $s_{T}$ is directly affected by the geometrical boundary conditions, which may well decrease the value of stability parameter $T o$. Such concerns are clearly not unprecedented: already Mason (1975) remarked the difficulty he experienced applying his theoretical predictions (based on parallel, linear isopycnals) to a real laboratory configuration of complex thermal profiles. The fact that the thermal gradient is neither monotonic nor uniform, may also lead to the development of localized baroclinic instability even if globally $T o>1$ can be assured, as indicated by Bastin and Read (1998). Moreover, the numerical results of Isachsen (2011), who also found extensive wave activity over a sloping terrain for $T o>1$, suggest that besides non-constant stratification, interior potential vorticity gradients, neglected by the Eady theory, can be responsible for such disagreements. Unfortunately with the experimentation techniques applied here it was not possible to obtain information directly on the thermal structure of the flow in the deeper regions or on potential vorticity.

Due to the aforementioned difficulties of comparison with theoretical predictions, we now restrict ourselves to discuss only the comparison of the results from the simulation-based stability analysis of von Larcher et al. (2013) and our experimental findings. To determine the regime boundaries of Fig. 3, von Larcher et al. (2013) have carried out a linear stability analysis relying on the spectral decomposition of the azimuthal modes, and thus, obviously could not resolve wave-wave interactions. However, as it is visible from our experimental data, the actual physical eigenmodes of the system are far not sinusoidal; single normal modes cannot be found but they usually show up in pairs or triplets. As an example, we refer to Figs. $2 \mathrm{~d}$ and $7 \mathrm{~b}$, which demonstrate that the spectral "fingerprint" of the 3-fold symmetric eigenmode also always contains the harmonic $m=6$, and a pattern that corresponds to a single $m=3$ Fourier mode per se simply cannot be excited. When the slope is present, this difference becomes critical, since in this configuration multiple physical eigenmodes can coexist that propagate at different drift rates, giving way to energy transfer between modes. Such wavewave interactions can either destabilize or stabilize the system; latter may be responsible for the disagreement between the numerical and experimental results.

The fact that sloping bottom topography leads to dispersive propagation of co-existing waves in a differentially heated rotating annulus was first noticed by Fultz and Kaynor (1959). By that time it was already well known, that in a rotating set-up over such a bottom end wall barotropic topographic Rossby waves may be excited (independently of the thermal boundary conditions, driven by the conservation of potential vorticity only). Therefore, it has been naturally assumed by Fultz and Kaynor (1959) that such waves are responsible for the observed behaviour as they modulate the waves of baroclinic instability. Because of the limitations of their measurement techniques, the results of the study are not exactly conclusive, yet they triggered further study of wave dispersion in similar set-ups. Pfeffer and Fowlis (1968) and Harlander et al. (2011) reported dispersive phenomena in the vicinity of the regime boundary between the "upper" axisymmetric and the regular wave flow regime and between two wave regimes, respectively, studying a flat bottom set-up with free surface. Occasionally, the very same apparatus that is investigated in the present paper - without slope but with free surface - also exhibited IV in the regime boundary region (besides the IB-type behaviour mentioned in Sect. 4.1), as noted by von Larcher and Egbers (2005) and Harlander et al. (2011).

In our set-up, a $\beta$ parameter can be defined as follows (Vallis, 2006):

$\beta=\frac{f \cdot \tan (\gamma)}{d_{\text {mid }}}$,

taken with $d_{\text {mid }}=10.85 \mathrm{~cm}$, the water depth at mid-radius $r_{\text {mid. }}$ In a two-layer model Mansbridge (1984) investigated the effect of different values of $\beta$ on the flow properties, and found that neighbouring modes can coexist if $\delta \equiv$ $\beta R o_{\mathrm{T}} / \sqrt{E k}$ is larger than a certain threshold (the value of which depends on mode number $m$, and was found to be between 10 and 63). Here the Ekman number $E k$ can be defined as $E k=v /\left(\Omega L^{2}\right)$, where $v \approx 10^{-6} \mathrm{~m}^{2} \mathrm{~s}^{-1}$ is the kinematic viscosity of water and the characteristic length was taken to be $L=r_{\text {smid }}=(a+b) / 2$. The values of $\delta$ in our sloping 
bottom runs were ranging from 340 to 950 (i.e. way over the aforementioned theoretical threshold) consistently with our detection of IV.

Further experimental studies are under consideration, involving direct velocity measurements applying the PIV technique to gain information on the flow field in the set-up. Using that data one could separate the propagation rate of the different baroclinic wave modes from the drift originating from the background flow. This will enable us to obtain dispersion relations and - in the case of strongly nonlinear patterns - amplitude-velocity relations which would lead to a deeper understanding of baroclinic waves in general, and would support a better comparison of the results to the predictions of different theories. We note that, assuming quasi-geostrophic connection between the time-averaged radial gradient of the observed surface temperature field and the zonal background flow, we already attempted to distinguish wave propagation from the flow. These trials however did not lead to any conclusive connection between the aforementioned quantities, which can be explained by the presence of multiple equilibria (i.e. that similar surface patterns may correspond to different flow states if the whole threedimensional water body is concerned). Therefore, PIV-based velocity measurements at different vertical levels would be crucial, as they may shed light on these issues as well.

Acknowledgements. The authors thank the two anonymous referees, and referee \#2 in particular for the significant contribution to this paper. We thank Yongtai Wang for his crucial help in performing the measurements. The fruitful discussions with WolfGerrit Früh and Kiril Alexandrov are also highly acknowledged. We are also grateful for the discussions with the members of the project group "Physical Mechanisms of Global Environmental Processes" (Budapest, Hungary, Grant number NK100296). This work has been funded by the German Science Foundation (DFG) and is part of the DFG priority program MetStröm (SPP 1276).

Edited by: H. J. Fernando

Reviewed by: two anonymous referees

\section{References}

Bastin, M. E. and Read, P. L.: A laboratory study of baroclinic waves and turbulence in an internally heated rotating fluid annulus with sloping endwalls, J. Fluid Mech., 339, 173-198, 1997.

Bastin, M. E. and Read, P. L.: Experiments on the structure of baroclinic waves and zonal jets in an internally heated, rotating, cylinder of fluid, Phys. Fluids., 10, 374-389, 1998.

Blumsack, S. L. and Gierasch, P. J.: Mars: The effects of topography on baroclinic instability, J. Atmos. Sci., 29, 1081-1089, 1972.

Eady, E. T.: Long waves and cyclone waves, Tellus, 13, 33-52, 1949.

Früh, W. G. and Read, P. L.: Wave interactions and the transition to chaos of baroclinic waves in a thermally driven rotating annulus, Phil. Trans. Roy. Soc. Lond. A, 355, 101-153, 1997.
Fultz, D. and Kaynor, R.: The propagation of frequency in experimental baroclinic waves in a rotating annular ring, The Rossby Memorial Volume, 359-371, New York, Rockefeller Institute Press, 1959.

Fultz, D., Long, R. R., Owebs, G. V., Bohan, W., Kaynor, R., and Weil, J.: Studies of thermal convection in a rotating cylinder with some implications for large-scale atmospheric motions, Meteor. Monogr., 4, 1-104, Am. Meteor. Soc., 1959.

Gyüre, B., Bartos, I., and Jánosi, I. M.: Nonlinear statistics of daily temperature fluctuations reproduced in a laboratory experiment, Phys. Rev. E, 76, 037301, doi:10.1103/PhysRevE.76.037301, 2007.

Harlander, U., von Larcher, Th., Wang, Y., and Egbers, C.: PIVand LDV-measurements of baroclinic wave interactions in a thermally driven rotating annulus, Exp. Fluids, 51, 37-49, 2011.

Harlander, U., Wenzel, J., Alexandrov, K., Wang, Y., and Egbers, C.: Simultaneous PIV and thermography measurements of partially blocked flow in a differentially heated rotating annulus, Exp. Fluids, 52, 1077-1087, 2012.

Hide, R.: An experimental study of thermal convection in a rotating fluid, Phil. Trans. Roy. Soc. Lond. A, 250, 441-478, 1958.

Isachsen, P. E.: Baroclinic instability and eddy tracer transport across sloping bottom topography: How well does a modified Eady model do in primitive equation simulations?, Ocean. Model., 39, 183-199, 2011.

Jánosi, I. M., Kiss, P., Homonnai, V., Pattantyús-Ábrahám, M., Gyüre, B., and Tél, T.: Dynamics of passive tracers in the atmosphere: laboratory experiments and numerical tests with reanalysis wind fields, Phys. Rev. E, 82, 046308, doi:10.1103/PhysRevE.82.046308, 2010

Lindzen, R. S., Farrel, B., and Jacqmin, D.: Vacillation due to wave interference: applications to the atmosphere and to annulus experiments, J. Atmos. Sci., 39, 14-23, 1982.

Lorenz, E. N.: The mechanics of vacillation., J. Atmos. Sci., 20, 448-464, 1963.

Mansbridge, J. V.: Wavenumber transition in baroclinically unstable flows, J. Atmos. Sci., 41, 925-930, 1984.

Mason, P. J.: Baroclinic waves in a container with sloping end walls, Phil. Trans. Roy. Soc. Lond. A, 278, 397-445, 1975.

Mechoso, C. R.: Baroclinic instability of flows along sloping boundaries, J. Atmos. Sci., 37, 1393-1399, 1980.

Pedlosky, J.: Geophysical fluid dynamics, Springer, New York, 1979.

Pfeffer, R. L. and Fowlis, W. W.: Wave dispersion in a rotating, differentially heated cylindrical annulus of fluid, J. Atmos. Sci, 25, 361-361, 1968.

Plumb, R. A.: The stability of small amplitude Rossby waves in a channel. J. Fluid Mech., 80, 705-720, 1977.

Read, P. L.: Applications of singular systems analysis to "baroclinic chaos", Physica D, 58, 455-468, 1992.

Rossby, C.-G.: On the dispersion of planetary waves in a barotropic atmosphere, Tellus, 1, 1-5, 1949.

Schreiber, T. and Schmitz, A.: Surrogate time series, Physica D, 142, 346-382, 2000.

Seelig, T., Harlander, U., Faulwetter, R., and Egbers, C.: Irregularity and singular vector growth of the differentially heated rotating annulus flow, Theor. Comput. Fluid Dyn., 27, 415-432, doi:10.1007/s00162-011-0255-5, 2012. 
Vallis, G. K.: Atmospheric and oceanic fluid dynamics - fundamentals and large-scale circulation, Cambridge University Press, Cambridge, 2006.

Vettin, F.: Über den aufsteigen Luftström, die Entstehung des Hagels und der Wirbel-Stürme, Ann. Physik Chemie, 102, 246$255,1857$.

von Larcher, Th. and Egbers, C.: Experiments on transitions of baroclinic waves in a differentially heated rotating annulus, Nonlin. Processes Geophys., 12, 1033-1041, doi:10.5194/npg-121033-2005, 2005. von Larcher, Th., Fournier, A., and Hollerbach, R.: The influence of a sloping bottom endwall on the linear stability in the thermally driven baroclinic annulus with a free surface, Theor. Comput. Fluid Dynami., 26, 433-451, doi:10.1007/s00162-012-0289-3, 2013.

Wordsworth, R. D., Read, P. L., and Yamazaki, Y. H.: Turbulence, waves, and jets in a differentially heated rotating annulus experiment, Phys. Fluids, 20, 126602-1-126602-12, 2008. 